\title{
Sustained Activity in Topographic Areas of Human Posterior Parietal Cortex during Memory-Guided Saccades
}

\author{
Denis Schluppeck, Clayton E. Curtis, Paul W. Glimcher, and David J. Heeger \\ Department of Psychology/Center for Neural Science, New York University, New York, New York 10003
}

In a previous study, we identified three cortical areas in human posterior parietal cortex that exhibited topographic responses during memory-guided saccades [visual area 7 (V7), intraparietal sulcus 1 (IPS1), and IPS2], which are candidate homologs of macaque parietal areas such as the lateral intraparietal area and parietal reach region. Here, we show that these areas exhibit sustained delay-period activity, a critical physiological signature of areas in macaque parietal cortex. By varying delay duration, we disambiguated delay-period activity from sensory and motor responses. Mean time courses in the parietal areas were well fit by a linear model comprising three components representing responses to (1) the visual target, (2) the delay period, and (3) the eye movement interval. We estimated the contributions of each component: the response amplitude during the delay period was substantially smaller $(<30 \%)$ than that elicited by the transient visual target. All three parietal regions showed comparable delay-period response amplitudes, with a trend toward larger responses from V7 to IPS1 and IPS2. Responses to the cue and during the delay period showed clear lateralization with larger responses to trials in which the target was placed in the contralateral visual field, suggesting that both of these components contributed to the topography we measured.

Key words: parietal cortex; cortical areas; fMRI; saccades; topography; human

\section{Introduction}

We can remember and discriminate visual stimuli that are separated in time by a delay period, a cognitive process known as working memory. Furthermore, we are able to maintain attention to a particular location of visual space over time and delay motor behaviors. Neural activity during such delay periods is believed to be a neural correlate of working memory, maintenance of attention, and the plan or intent to move. Such sustained delay-period activity has been reported in both human and monkey prefrontal, inferotemporal, and parietal cortex (Andersen and Buneo, 2002; Goldberg et al., 2002; Curtis and D'Esposito, 2003; Glimcher, 2003; Passingham and Sakai, 2004).

In this study, we characterized the response properties of three cortical areas in human posterior parietal cortex [visual area 7 (V7), intraparietal sulcus 1 (IPS1), and IPS2] during memoryguided saccades, a well studied delayed oculomotor behavior. The cortical areas were identified in each subject by mapping the topographic organization for delayed saccades to reveal areas containing neurons with properties like those in the macaque areas lateral intraparietal area (LIP) and parietal reach region (PRR) (Sereno et al., 2001; Connolly et al., 2003; Schluppeck et al., 2005). Each of the three areas contained topographic maps of the contralateral visual-saccadic space. One of the areas corre-

Received Dec. 14, 2005; revised March 29, 2006; accepted April 5, 2006.

This research was supported by National Institute of Mental Health Grant R01-MH69880 and the Seaver Foundation. We thank Justin Gardner, Jonas Larsson, and Shani Offen for helpful discussion and comments on this manuscript.

Correspondence should be addressed to Denis Schluppeck, Department of Psychology/Center for Neural Science, 4 Washington Place, New York University, New York, NY 10003. E-mail: ds@cns.nyu.edu.

D01:10.1523/JNEUROSCI.5330-05.2006

Copyright $\odot 2006$ Society for Neuroscience $\quad$ 0270-6474/06/265098-11\$15.00/0 sponded to visual area V7, as determined by standard retinotopic measurements. The other two, labeled IPS1 and IPS2 for their anatomical positions, were located on the medial aspect of the intraparietal sulcus, rostral to V7 at the transverse occipital sulcus. The topographic mapping experiment, in which subjects made memory-guided saccades to a series of target locations, extracted a single topographic organization for a number of possible separable factors, including transient presentation of the target, transient (exogenous) attention to the target presentation, transient motor activity involved in making the saccade, sustained motor intention, sustained (endogenous) attention to the cued location, sustained spatial working memory for the location, and advance preparatory processes that might be occurring because of the predictability of the upcoming target location. Some or all of these confounding factors could have contributed to the measured topographic maps. Importantly, we could not, from that experiment, conclude that the candidate areas exhibited sustained delay-period activity. Because the delay period in that experiment was constant and short ( $3 \mathrm{~s}$ ) compared with the dispersion of hemodynamic responses, it was not possible to unambiguously attribute the measured cortical activity to the delay periods.

We therefore performed an experiment, reported here, to explicitly test for sustained delay-period activity during memoryguided saccades in the predefined areas (V7, IPS1, and IPS2). We confirmed that all three topographic areas of human posterior parietal cortex exhibited sustained delay-period activity, a critical physiological signature of the potentially homologous areas in the macaque brain (including LIP and PRR). The amplitudes of the sustained delay-period responses, although statistically significant, were small compared with transient responses at the 
beginning (visual target presentation) and end (eye movement interval) of each trial, similar to electrophysiological results in monkey parietal cortex. Both the cue and delay-period responses were lateralized, suggesting that both of these components contributed to the topography observed in the previous mapping experiments.

\section{Materials and Methods \\ Subjects}

Four experienced subjects participated in this study with written consent. Procedures were in compliance with the safety guidelines for magnetic resonance imaging (MRI) research and approved by the human subjects Institutional Review Board at New York University. Each subject participated in a number of scanning sessions: one session to obtain a highresolution anatomical volume, two sessions to measure the retinotopic maps in visual cortex (see below), two to three sessions to measure topographic organization of posterior parietal cortex, and two to three sessions to measure delay-period activity.

\section{Visual stimuli and task}

Stimuli were presented via a liquid crystal display projector and longthrow optics onto a back-projection screen in the bore of the MR scanner. Subjects were supine and viewed the projected stimuli (maximum eccentricity, $14^{\circ}$ ) through an angled mirror.

Main experiment (delayed saccades). Subjects fixated centrally while a peripheral target was briefly $(\sim 100 \mathrm{~ms})$ presented. After a variable delay (3-15 s in $1.5 \mathrm{~s}$ increments) the fixation spot disappeared, cueing subjects to make a saccade to the remembered target location (Fig. 1A,B). After the saccade, the target reappeared, providing feedback. Subjects were instructed to make a corrective saccade, if necessary, to accurately refixate the target. After an additional $1.5 \mathrm{~s}$ delay, the central fixation spot reappeared, signaling the subject to fixate that target with a return saccade. The next trial began after a randomized intertrial interval (10.5-15 $\mathrm{s}$ in $1.5 \mathrm{~s}$ increments). Brightening of the fixation spot cued the impending start of the next trial. Subjects were instructed to make saccades as quickly as possible after the cue. Targets appeared at 1 of 20 locations at $8-11^{\circ}$ eccentricity within $45^{\circ}$ of the horizontal meridian (Fig. 1C). The order of trials was pseudorandom such that subjects could not predict the target location or delay-period duration before the onset of each trial. We included a larger number of trials with medium to long delay periods because they are more informative for estimating sustained delay-period activity (Fig. $1 D$ ). We also included control trials to measure the functional MRI (fMRI) response to the presentation of the visual target alone, in which the delay period was aborted after $1.5 \mathrm{~s}$ (fixation turned red) and no eye movements were made. Subjects performed the task in a series of scans lasting $300 \mathrm{~s}$ and were allowed to rest for several seconds between scans. Data from all trials were included in the subsequent analyses. To ensure that subjects could fixate throughout the duration of the delay period, we measured eye position during the task inside the scanner for one subject (infrared video eye tracker, ASL 504LR; Applied Science Laboratories, Bedford, MA). Additionally, we confirmed in another group of 12 subjects performing the same task that the accuracy of the memory-guided saccades did not depend on delay-period duration for delays between 7.5 and $13.5 \mathrm{~s}$ (supplemental Fig. 1, available at www. jneurosci.org as supplemental material), consistent with previous reports (White et al., 1994).

Retinotopy. Retinotopic visual areas (V1, V2, V3, V3A, V3B, hV4, and V7) were defined using standard methods (Engel et al., 1994, 1997; Sereno et al., 1995; DeYoe et al., 1996). For details, see Schluppeck et al. (2005).

Topography for delayed saccades. Subjects performed a delayed saccade task in which targets appeared at successive locations "around the clock" at the same eccentricity, separated by $30^{\circ}$ angular steps (Sereno et al., 2001; Schluppeck et al., 2005). On each trial, a small random angular offset was added to the target locations $\left(\right.$ range, $\pm 5^{\circ}$ ). Each cycle lasted $54 \mathrm{~s}$ (number of target locations $\times$ trial duration $=12 \times 4.5 \mathrm{~s}$ ). Subjects performed the task for five cycles, resulting in scans lasting $270 \mathrm{~s}$. We alternated scans in which the target progressed in clockwise and coun-

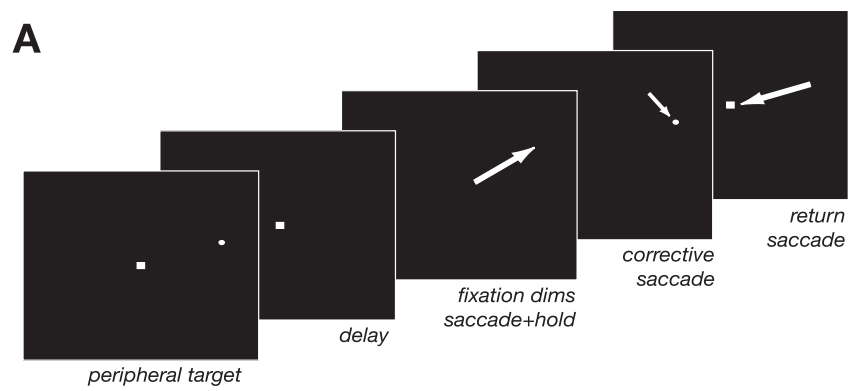

B

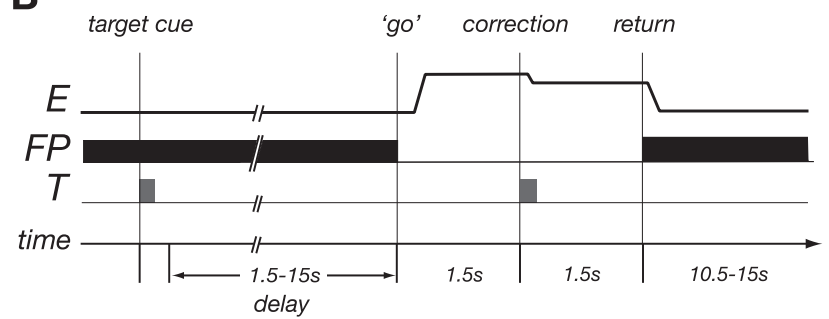

C

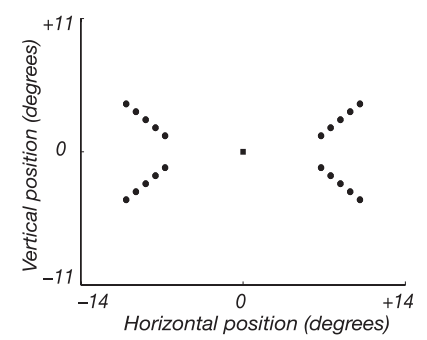

D

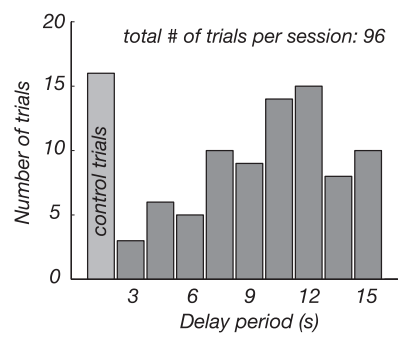

Figure 1. Stimulus and behavioral task. $\boldsymbol{A}, \boldsymbol{B}$, Subjects were instructed to fixate centrally while a peripheral target was briefly ( $\sim 100 \mathrm{~ms})$ presented at one of 20 locations. Subjects remembered the location of the target during a variable delay period (3-15 s). Dimming of the fixation spot signaled the subjects to make a saccadic eye movement to and hold gaze at the remembered target location. The target then reappeared, allowing for a corrective saccade that subjects had been instructed to make. On brightening of the central fixation spot 1.5 s later, subjects made a return saccade. The next trial began after a variable interval (10.5-15 s). $\boldsymbol{B}$, Timing of each event during a trial. E, Eye position; $F$, fixation point; $T$, target. $C$, Targets were presented at 1 of 20 locations close to the horizontal meridian. D, Delay-period durations were $3-15 \mathrm{~s}$ in increments of $1.5 \mathrm{~s}$. The histogram shows the number of trials per delay period for each scanning session. We included control trials that were aborted after $1.5 \mathrm{~s}$ (fixation turned red) and no eye movements were made. The order of trials (target, delay) was pseudorandomized.

terclockwise directions and allowed subjects to rest for several seconds between scans.

In each topography scanning session, we also included one or two scans (duration, $270 \mathrm{~s}$ ), in which subjects alternated between blocks of central fixation (13.5 s) and blocks consisting of a rapid series (one every $750 \mathrm{~ms}$ ) of eye movements (13.5 s). During the eye movement block, subjects shifted their gaze between the central fixation spot and a series of peripheral targets. The presentation of the peripheral targets $\left(750 \mathrm{~ms}, 10^{\circ}\right.$ eccentricity, random angles) alternated with the central fixation spot (750 ms), only one of which was visible at any time.

We defined regions of interest (ROIs) corresponding to areas V7, IPS1, and IPS2 (see Fig. 2) based on the results from the topography protocol and further restricted each ROI to subregions of cortical gray matter that exhibited strong responses in the saccade-versus-fixation condition (for details, see Schluppeck et al., 2005). As reported previously, there were three topographically organized regions on the medial aspect of the intraparietal sulcus (Schluppeck et al., 2005). All three areas were evident in both hemispheres of all four subjects. One of the parietal regions corresponded to area $\mathrm{V} 7$, as confirmed by standard retinotopic mapping. We previously labeled the other areas IPS1 and IPS2 for their anatomical location, specifically to avoid drawing a premature inference about possible homologies with macaque cortical areas. Data from the main exper- 
iment were analyzed by averaging over regions of cortical gray matter corresponding to each of the three topographically defined cortical areas. Additionally, we defined a region of interest just lateral to the three topographic areas, which did not show topographic organization either for delayed saccades or during conventional retinotopic mapping.

\section{Functional imaging}

We used functional magnetic resonance imaging at $3 \mathrm{~T}$ (Allegra; Siemens, Erlangen, Germany) to measure blood-oxygen level-dependent (BOLD) changes in cortical activity. During each fMRI scan, a time series of volumes was acquired using a $\mathrm{T} 2{ }^{*}$-sensitive echo planar imaging pulse sequence (repetition time, $1500 \mathrm{~ms}$; echo time, $30 \mathrm{~ms}$; flip angle $75^{\circ} ; 24$ slices; $3 \times 3 \times 3 \mathrm{~mm}^{3}$ voxels; field of view, $192 \mathrm{~mm}$ ). Images were acquired using custom radio frequency coils (NM-011 transmit headcoil and NMSC-021 four-channel phased array receive coil; NOVA Medical, Wakefield, MA).

\section{Data analysis}

To minimize head motion, subjects were stabilized by use of a bite bar and/or foam padding. Post hoc image registration was used to correct for residual head motion [MCFLIRT (motion correction using FMRIB's Linear Image Registration Tool)] (Jenkinson et al., 2002). Data from the first $14 \mathrm{~s}$ of each fMRI scan were discarded to minimize transient effects of magnetic saturation. Additional preprocessing of the fMRI data were as follows. First, we bandpass filtered the time series at each voxel to compensate for the slow drift typical in fMRI measurements (Biswal et al., 1995, 1997a,b; Zarahn et al., 1997; Purdon and Weisskoff, 1998; Smith et al., 1999). The cutoff frequencies were 0.01667 and $0.1667 \mathrm{~Hz}$, making the low-frequency cutoff much lower than the longest delay period to ensure that the filter did not attenuate the sustained delayperiod activity. Next, we divided the time series of each voxel by its mean intensity to convert the data from arbitrary image intensity units to percentage signal modulation (and to compensate for the decrease in mean image intensity with distance from the receive coil). The preprocessed time series from the main (delayed saccade) experiment were averaged over a region of cortical gray matter corresponding to each ROI, as defined topographically.

We then performed two complementary analyses of data to estimate (1) the fMRI response time course for each trial type, and (2) the response amplitudes for each of the three intervals of the behavioral task (visual target presentation, delay period, and eye movement interval). The data were analyzed separately for each of the predefined cortical areas.

fMRI responses. We used standard event-related analysis methods ( $\mathrm{Bu}-$ rock et al., 1998; Dale, 1999; Burock and Dale, 2000) to estimate the fMRI response time course for each trial type. There were 10 different trial types: nine corresponding to different delay-period durations $(3-15 \mathrm{~s}$ in increments of $1.5 \mathrm{~s}$ ) and one corresponding to a control, during which no eye movements were made (see above, Visual stimuli and task). Specifically, we calculated the fMRI responses from each scan by trial-triggered averaging, solving an equation of the form $\mathbf{y}=A \mathbf{x}$, where the vector $\mathbf{y}$ was the measured time course during one scan (200 time points), the vector $\mathbf{x}$ contained the estimated fMRI responses for a given trial type in that scan, and $A$ was the design matrix that characterized the trial sequence. The design matrix $A$ had $k$ rows ( $k$ indicates the number of time points in a scan, 200) and $n$ columns ( $n$ indicates the number of time points in the estimated response time courses for trial type). The first column of $A$ contained the value 1 at indices corresponding to the onset of trials with a given delay-period duration, 0 elsewhere. The second column contained a 1 at indices corresponding to the second time point of those trials, and so on. We calculated the ordinary least-squares solution as $\mathbf{x}=$ $\left(A^{T} A\right)^{\dagger} A^{T} \mathbf{y}$, where []$^{T}$ is matrix transpose and []$^{\dagger}$ is pseudoinverse. We repeated this calculation for all trial types.

The resulting fMRI responses from this analysis were further averaged across 8-16 scans and plotted with error bars representing the SEM across these $8-16$ repeats. We first combined data across all target locations; in a subsequent analysis step, we analyzed the effect of target location (see below, Lateralization). Figure 3 shows example fMRI responses from one region of interest (in one subject) for trials with different delayperiod durations.
Response amplitudes. We used multiple linear regression to estimate the amplitudes of the responses to each of the three distinct intervals of the memory-guided saccade task. This was done by constructing a linear model of the underlying neural activity for each $300 \mathrm{~s} \mathrm{scan}$ (see Fig. 4). Each trial was modeled with three components: (1) a transient at the beginning of each trial (an impulse at $t=0$, labeled $v$ ) that reflected the onset of the visual target, (2) a sustained component that had a constant amplitude and lasted throughout the delay period (duration of $3 \mathrm{~s}-15 \mathrm{~s}$, labeled $d$ ), and (3) a transient component at the end of each trial that captured the saccade to the remembered target, the reappearance of the target, and the corrective and return saccades (an impulse at the time of fixation dimming, labeled $s$ ). This is illustrated for one example trial in Figure $4 \mathrm{~A}$. Each of the components $(v, d$, and $s$ ) of the neural model was then convolved with a model of the hemodynamic impulse response function and bandpass filtered in the same way as the measured fMRI data to yield three predictors of the fMRI measurements. Linear regression (as written above) was used to estimate the three response amplitudes that, when multiplied by the three predictors, yielded the best (least-squares) fit to the data.

The hemodynamic impulse response function was modeled with a difference of two gamma functions (Glover, 1999; Jezzard et al., 2003):

$$
H(t)=\left[\frac{t}{d_{t}}\right]^{a_{1}} \exp \left[\frac{-\left(t-d_{1}\right)}{b_{1}}\right]-c\left[\frac{t}{d_{2}}\right]^{a_{2}} \exp \left[\frac{-\left(t-d_{2}\right)}{b_{2}}\right],
$$

where $d_{i}=a_{i} b_{i}$ is the time-to-peak. The parameters used were $a_{1}=5.15$, $a_{2}=16.26, b_{1}=0.97, b_{2}=0.94$, and $c=0.09$, which makes the shape of the hemodynamic response function similar to that used by other standard analysis packages (Friston et al., 1995).

We fit the model, separately for each scan (200 time points), to obtain estimates for the three amplitudes $(v, d$, and $s)$. Because we randomized both the order of the trial types and intertrial intervals, this randomization (in addition to measurement noise) contributed to the variability across scans in the estimated response amplitudes. To assess the variability in the model fits and to compare the model with the measured fMRI responses, we calculated the mean and SD of the model fit using the trial-triggered averaging analysis outlined above. The mean and SD of the model fit was then superimposed with the mean and SEM of the fMRI responses (see Fig. 3). To quantify the model fit across ROIs and subjects, we calculated the proportion of variance accounted for in the observed mean fMRI responses $\left(r^{2}\right)$ :

$$
r^{2}=1-\frac{\operatorname{var}(\text { residual })}{\operatorname{var}(\text { observed })} .
$$

We also fit the model omitting each of the three components $(v, d$, and s) in turn. Predictably, decreasing the number of parameters led to worse fits and therefore a decrease in the variance accounted for by the model. For example, the effect of omitting the sustained activity during the delay period from the fit (equivalent to assuming it to be 0 ) is illustrated in supplemental Figure 2 (available at www.jneurosci.org as supplemental material): the fit systematically underestimated the sustained activity during the delay period. (The model was fit simultaneously for trials at all delay-period durations.) Assessing the statistical significance of such a worsening of the fits, however, is complicated. Standard statistical tests on nested hypotheses (e.g., $F$ tests for the difference in the variance of residuals) require assumptions about the independence of residuals (the difference between the measured fMRI response and the model fit) at consecutive time points. These statistical assumptions are likely to be false and therefore care has to be taken in interpreting the resulting $p$ values; we also relied on visual inspection that clearly indicated that the model fits were substantially worse after omitting any of the three components. We also performed a complementary statistical test (that did not rely on statistical independence at consecutive time points) to demonstrate that each of the three components was significantly greater than 0 (see Results and Table 2).

Lateralization. Because our topographic mapping experiments (Schluppeck et al., 2005) revealed contralateral representations of the visual field in areas V7, IPS1, and IPS2, we expected one or more components of the measured fMRI responses to show larger amplitudes for 
trials in which targets appeared in the contralateral visual field, e.g., trials in the left visual hemifield for right hemisphere ROIs. We therefore fit the model (see above) for trials left and right of fixation separately to the responses measured in left and right hemisphere V7, IPS1, and IPS2. To assess whether components of the fMRI responses were lateralized we calculated a lateralization index, $L$, on the estimated amplitude parameters as

$$
L=\frac{\beta_{\text {contra }}-\beta_{\text {ipsi }}}{\left|\beta_{\text {contra }}\right|+\left|\beta_{\text {ipsi }}\right|},
$$

where $\beta_{\text {contra }}$ is the estimated response amplitude for contralateral trials, and $\beta_{\text {ipsi }}$ is the estimated response amplitude for ipsilateral trials. The lateralization index is positive $(0<L \leq 1)$ if responses are larger for contralateral trials, negative $(-1 \leq L<0)$ if responses are larger for ipsilateral trials, and 0 if responses are equal for both ipsilateral and contralateral trials.

Statistical parameter mapping. In addition to the region-of-interest analyses described below, we also performed conventional statistical parameter mapping (Friston et al., 1991) on a subset of the data (Fig. 2 shows data from subject 3 , one scan, $300 \mathrm{~s}$ ). This analysis allowed us to localize regions of cortex that exhibited statistically significant sustained delay-period activity, even if they did not exhibit topography. Analysis was performed using FEAT (FMRI Expert Analysis Tool) version 5.4, part of FSL [Software Library of FMRIB (Functional Magnetic Resonance Imaging of the Brain), www.fmrib.ox.ac.uk/fsl]. The general linear model was identical to that outlined above, with three covariates or explanatory variables: one for transient events at the beginning $(v)$ of every trial, one for transient events at the end $(s)$ of every trial, and one to account for sustained responses during the delay period $(d)$. Statistical maps were thresholded using clusters determined by $Z>2.3$ and a (corrected) cluster significance threshold of $p=0.01$ (Worsley et al., 1992). The resulting thresholded statistical maps were rendered on a partially inflated cortical surface representation of the subject's cortex using custom software (Larsson, 2001).

\section{Results}

\section{Topography and statistical parameter mapping}

We defined three regions in posterior parietal cortex (V7, IPS1, and IPS2) in each subject by topographic mapping as described previously (see Materials and Methods) (Schluppeck et al., 2005). For additional analysis, data from the main experiment were then analyzed by averaging over regions of cortical gray matter corresponding to each of the three topographically defined cortical areas. To confirm the choice of our ROIs, we also performed conventional statistical parameter mapping on a subset of the data (Fig. 2). This analysis revealed regions in the intraparietal sulcus with statistically significant sustained responses during the delay periods, which fell within the ROIs defined by topographic mapping.

\section{fMRI responses to memory-guided saccades}

The fMRI responses exhibited three distinct components corresponding to the three intervals in the memory-guided saccade task (Fig. 3). (1) The first component was locked to the onset of the trial when the visual stimulus (cue) was presented. (2) The second component reflected a sustained response lasting throughout the delay period. (3) The third component was locked to the eye movement interval at the end of each trial. By systematically varying the delay-period duration, we unambiguously determined that activity was sustained during the delay periods in all three areas. For longer delay periods (e.g., 12 and $15 \mathrm{~s})$ the transient events at the beginning and end of the trials were separated from one another in time, despite the relatively slow hemodynamics. The measured fMRI responses did not return to baseline during the delay periods, even for the longest

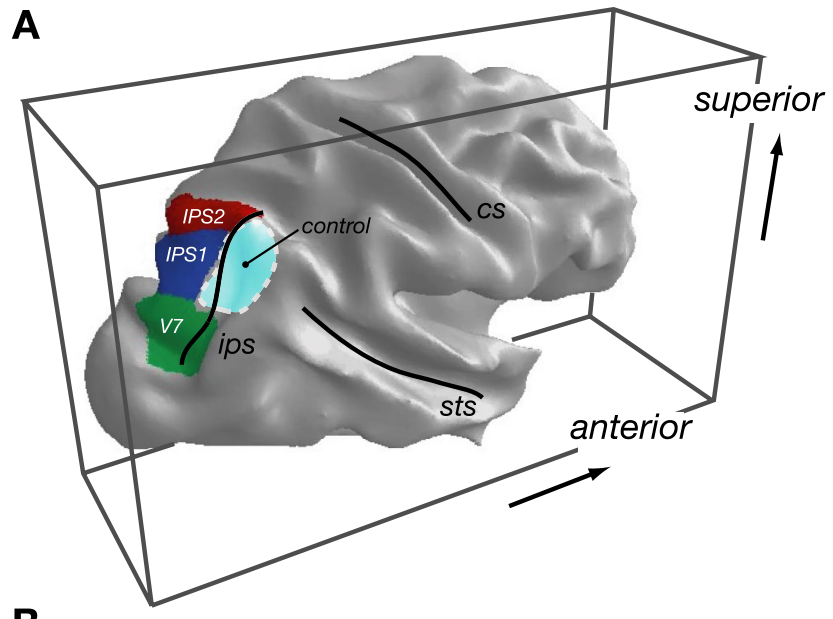

B
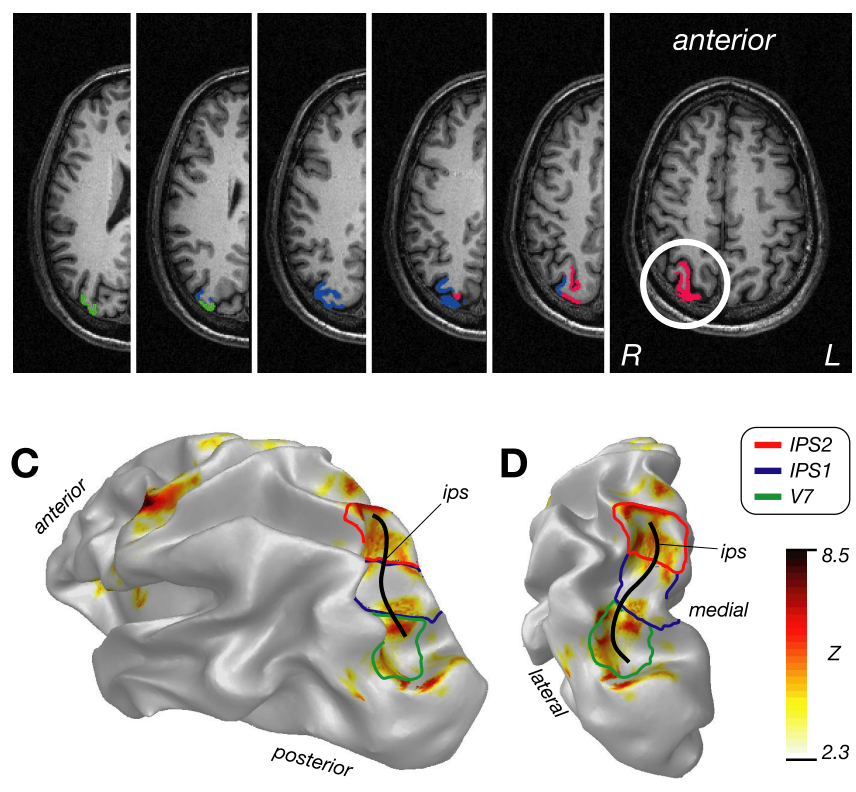

Figure 2. Cortical areas in posterior parietal cortex. $A$, Tessellated surface representation of the gray/white matter boundary in lateral/posterior aspect (right hemisphere of one subject, partially inflated). Three cortical areas are indicated. Green, V7; dark blue, IPS1; red, IPS2. These cortical areas were defined in a separate experiment that measured topographic organization during delayed saccades (Schluppeck et al., 2005). Light blue, A region within the IPS adjacent to IPS1 and IPS2, approximately comparable in size but that did not exhibit topography. This more lateral IPS region was used as an internal control. cs, Central sulcus; sts, superior temporal sulcus. B, Axial slices of the high-resolution anatomical image of the same subject. (Radiological convention; spacing between displayed slices, $4 \mathrm{~mm}$.) Cortical areas are superimposed in color. L, Left; R, right. C, D, Statistical parameter mapping. Partially inflated left hemisphere (subject S3) in lateral $(\boldsymbol{C})$ and posterior $(\boldsymbol{D})$ aspect. Colors, Areas with significant delay-period activity ( $Z>2.3$; corrected cluster significance threshold, $p<0.01$; see Materials and Methods). Region of interest outlines: green, V7; blue, IPS1; red, IPS2. Location of intraparietal sulcus (ips) is indicated as an anatomical landmark.

delay-period durations. For short delay periods (e.g., 3 and $6 \mathrm{~s}$ ), conversely, the different components of the response coalesced because of the sluggishness of the hemodynamics.

\section{Model simulations}

The slow timescale of the hemodynamic response presents a challenge for fMRI measurements aimed to unambiguously separate sensory, motor, and delay-period activity (Fig. 4). We performed model calculations to illustrate this point and to optimize the design of our imaging experiment. We calculated the expected 

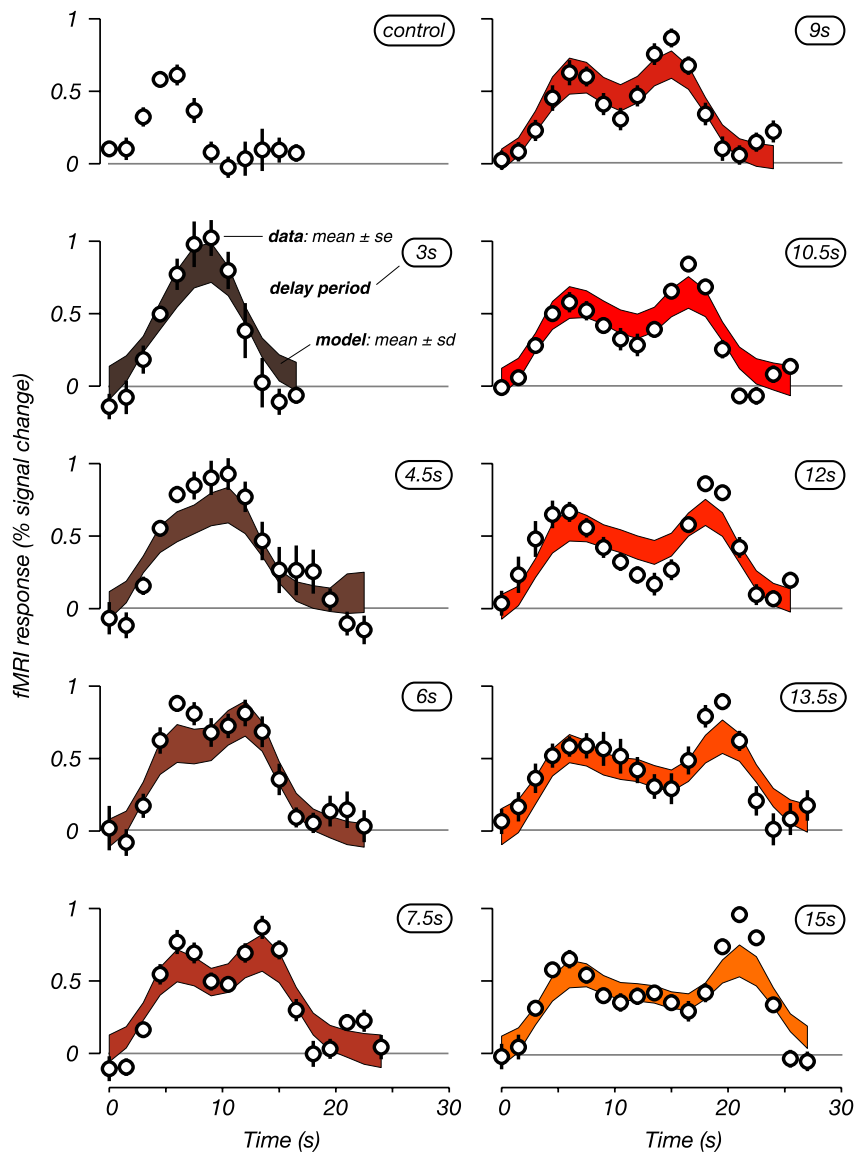

Figure 3. Data and model fits (IPS2, right hemisphere, S1). Open symbols and error bars indicate mean fMRI response \pm 1 SEM across $12-16$ scans. Top left panel, fMRI response for control condition (trials aborted after $1.5 \mathrm{~s}$, no eye movements). Other panels, fMRI responses for each delay-period duration. Shaded region indicates \pm 1 SD of model fits (see Materials and Methods). Estimated response amplitudes for the full model fit: $v=1.22, d=0.28, s=1.44$, $r^{2}=0.77$.

fMRI response for single trials by adopting a model of the underlying neural activity and a model for how an fMRI measurement depends on underlying neural activity. The model of neural activity had three components: (1) a transient at the beginning of the trial, $v$ (visual), that reflected the onset of the visual target, (2) a sustained component, $d$ (delay), that had a constant amplitude and lasted throughout the delay period, and (3) a transient component at the end of each trial, $s$ (saccade), that captured the eye movement interval (Fig. 4A). This model for the time course of neural activity was convolved with a hemodynamic impulse response function to yield predicted fMRI responses (Fig. $4 B$ ). The simulation results illustrate two critical issues.

First, because the hemodynamic response acts as a leaky integrator, sustained neural activity had a large effect on the simulated fMRI responses (Fig. 4C). We calculated the predicted fMRI response for two trials that only differed in the amplitude of the delay-period activity. For the sake of illustration, the amplitudes of the transient events at the beginning $(v)$ and end $(s)$ of the delayed-saccade trial were set to 1 (arbitrary units). The duration of the delay period was set to $15 \mathrm{~s}$, and the amplitude of the delay-period activity $(d)$ was set to either 0.1 or 0.3 . The two resulting fMRI responses illustrate the disproportionate effect of sustained neural activity. The ratios between the initial peak values and the local minimum during the delay period were $\sim 0.1$ : $0.35-0.29$ and 0.3:0.45-0.67 for the two different levels of delay- period activity compared with $0.1: 1$ and $0.3: 1$, the ratios in the model of neural activity.

Second, for short delay-period durations, the simulated fMRI responses to the onset of the trial were confounded with changes in the amplitude of delay-period activity (Fig. $4 D$, insets show simulated neural responses). Likewise, the simulated fMRI response at the end of the trial was subject to the same confound when the activity during the eye movement interval was superimposed with the remaining effects of the delay-period activity. For long delay periods, conversely, the responses to the visual cue and the delay period could be unambiguously resolved (Fig. 4E).

We therefore adopted an experimental protocol that included long delay-period durations (Fig. 1). Although long delay trials are most informative for estimating sustained activity, short delay-period trials were included as well to control subjects' behavior. In fact, the delay-period duration was variable and randomized (from 3 to $15 \mathrm{~s}$ in increments of $1.5 \mathrm{~s}$ ), and the target location was also randomized (see Materials and Methods). Subjects were instructed to hold fixation until cued to move at the end of each trial and then to make saccades as quickly and as accurately as possible. Each subject practiced the task extensively before scanning. To ensure that subjects held fixation accurately throughout the duration of the delay period, we measured eye position during the task inside the scanner in one of our subjects (supplemental Fig. 1, available at www.jneurosci.org as supplemental material). Fixation error was $\sim 1^{\circ}$ on average, much less than eccentricity of the saccade targets, and inspection of the eye position traces revealed no evidence of large saccades during the delay periods. In a separate series of related experiments (Srimal et al., 2005), we measured eye movements on 12 subjects performing the same task while in the scanner and found that the saccade accuracy did not depend on delay-period duration for delays between 7.5 and $13.5 \mathrm{~s}$ (supplemental Fig. 1, available at www.jneurosci.org as supplemental material), a result that confirms previous psychophysical studies of saccades at long delays (White et al., 1994). The variable, randomized delays motivated subjects to prepare to make a saccade and to maintain the intended eye movement during the delay period. With a fixed, predictable delay period, subjects might have used a strategy in which they would not need to hold the motor plan in a "ready to go" state. The randomized target locations minimized the possibility that subjects learned to make stereotyped eye movements. With fixed, predictable target locations, subjects might have overlearned the eye movements, relying on different neural circuitry.

\section{Estimating response amplitude of sustained delay-period activity}

We fit the measured fMRI responses with a linear model comprising three components identical to those described above. Note that there were only three free parameters: the amplitudes of three components, one corresponding to the sustained response during the delay period, and the other two to the transient responses at the beginning and end of each trial.

The linear model based on these three components provided an excellent fit to the data (Fig. 3, Table 1). Because the order of trials was randomized, each trial was preceded by a different history of trials, which contributed (along with measurement noise) to the variability in the model fits. To illustrate this variability, we computed the mean and SD of the model fits (see Materials and Methods) and plotted them superimposed with the measured fMRI responses. The quality of the model fits was quantified by comparing the resulting mean model fits with the mean fMRI 
A

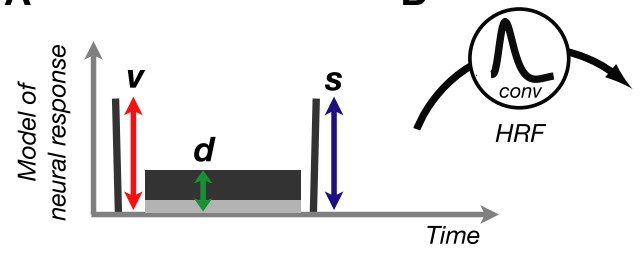

D

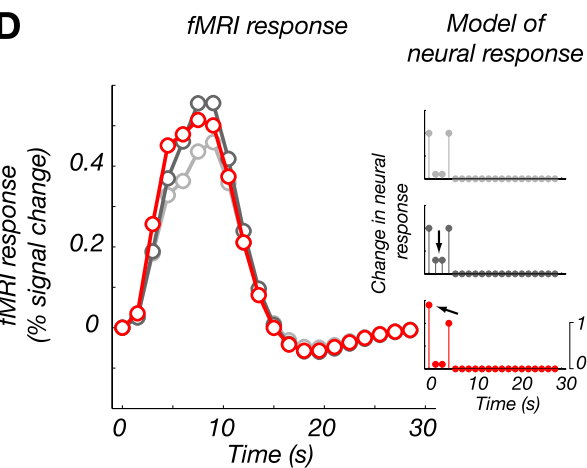

C

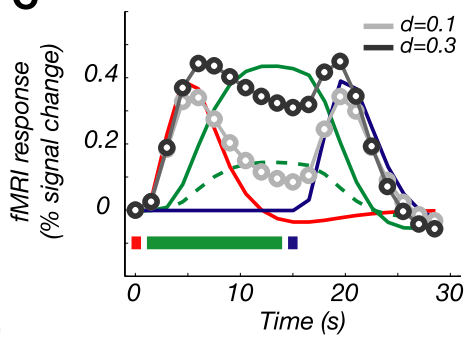

E

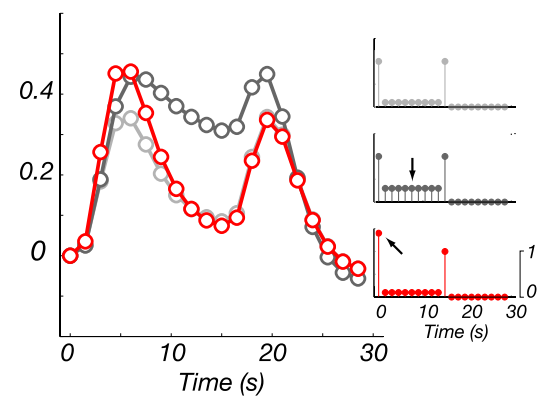

Figure 4. Model simulations. $\boldsymbol{A}$, Three response amplitude parameters defined the model of neural response during a given trial: $v$, visual target (transient response at the beginning of each trial); $d$, delay period; $s$, saccades (transient at the end of trial). Delay-period response amplitudes (light gray, 10\%; dark gray, 30\%) specified relative to the visual onset response amplitude. $\boldsymbol{B}$, Predicted fMRI responses were calculated by convolving (conv) the neural response model with a hemodynamic impulse response function (HRF) (difference of two gamma functions, two free parameters, delay and dispersion; see Materials and Methods). $\boldsymbol{C}$, Predicted fMRI response time courses. Gray curves and symbols (open circles), Responses with low (10\%, light gray) and high (30\%, dark gray) levels of delay-period activity. Thin red, green, and blue curves show contributions of each component $(v, d$, and s) to the total predicted fMRI responses. Red, $v$; green, $d$ (dashed line, low; solid line, high); blue, s. Colored bars, Corresponding timing of the three components before convolution. $\boldsymbol{D}, \boldsymbol{E}$, Simulated responses for short $(\boldsymbol{D})$ and long $(\boldsymbol{E})$ delay periods. Colors, Responses with low (10\%, light gray) and high (30\%, dark gray) levels of delay-period activity. Red line, Responses with increased amplitude for the visual transient $(+40 \%)$ that are confounded with sustained activity at short but not long delay-period durations. Insets, Simulated neural responses over time (before convolution with hemodynamic response function). For short delays, amplitude variations in delay period and visual transient signals are confounded in the fMRI measurements.

Table 1. Model fit parameters

\begin{tabular}{lllll}
\hline Region of interest & $v$ & $d$ & $s$ & $r^{2}$ \\
\hline V7 & $0.79 \pm 0.27$ & $0.12 \pm 0.09$ & $2.02 \pm 0.43$ & 0.71 \\
IPS1 & $0.72 \pm 0.28$ & $0.16 \pm 0.08$ & $1.67 \pm 0.51$ & 0.72 \\
IPS2 & $0.91 \pm 0.24$ & $0.25 \pm 0.07$ & $1.78 \pm 0.25$ & 0.72 \\
\hline
\end{tabular}

Best-fit parameters across subjects and hemispheres (mean and SEM across subjects). $r^{2}$ column indicates the variance accounted for by the three-parameter model, averaged across subjects.

responses. Although there were only three free parameters, the model accounted on average for $>70 \%$ of the variance in the data (Table 1). Leaving out any of the three fit parameters decreased the variance accounted for by the model substantially (supplemental Fig. 2, available at www.jneurosci.org as supplemental material).

There were small discrepancies between the model fits and the measured fMRI responses. For the fMRI responses from the subject shown in Figure 3, for example, the model fits did not exactly capture the transient changes at the beginning and end of the measured fMRI responses $\left(r^{2}=0.77\right.$ for this example). There are several possible explanations for these small discrepancies. First, we assumed a certain shape for the hemodynamic response function (double gamma), which is known to vary across different regions of the brain and across subjects. We explored whether the particular choice of parameters for the shape of the hemodynamic response function caused these discrepancies by changing them and refitting the data. None of these changes in parameters, however, improved the overall fit of the model. Second, because only three parameters were used to fit the data across repeated scans, the model captured only the average response amplitudes of the components across all delayperiod durations. This reflects our assumption that the neural processes underlying the neural responses are constant throughout the delay period for each delay-period duration. The model fit in Figure 3 appears to systematically underestimate the BOLD signal time course during short delays and overestimate it for longer delays (e.g., compare 4.5 vs $12 \mathrm{~s}$ delays). This might suggest that the amplitude of the responses decreased with delay-period duration, but this effect was not apparent across subjects and ROIs, and it would be difficult to quantify given the small amplitudes (see below and Table 1) of the delay-period responses. An extended model that allowed the responses to vary over time, allowing for a linear decrease during the delay period or with different values for each increment in delayperiod duration, would fit the data better, however, at the cost of including more parameters and with the concomitant risk of overfitting the data.

We confirmed that all three topographic areas of human posterior parietal cortex exhibited sustained delay-period activity (Fig. 5, Tables 1, 2). The delayperiod response amplitudes were significantly larger than 0 in IPS 2 in all four subjects and in V7 and IPS1 in three of four subjects (Table 2). Even for the subject in which there was no statistically significant delay-period activity in bilateral V7 and IPS1, the left hemisphere ROIs showed statistically significant sustained responses for contralateral trials. Although statistically significant, delay-period response amplitudes were small compared with the transient responses corresponding to the visual stimulus and the eye movement intervals (Table 1). Note, however, that the relatively small amplitude of the delay-period activity could still affect the shape of the observed fMRI responses substantially, because the hemodynamic response function acts, in effect, as a leaky integrator over time (Fig. 4). As an internal control, we defined a region within the IPS adjacent to IPS1 and IPS2, approximately comparable in size but that did not exhibit topography, neither during delayed saccades nor during conventional retinotopy measurements. This control IPS region did not exhibit sustained, delay-period activity (Fig. 5C, inset).

\section{Lateralization}

Because our topographic mapping experiments (Schluppeck et al., 2005) revealed contralateral representations of the visual field in areas V7, IPS1, and IPS2, we expected one or more components of the measured fMRI responses to show larger amplitudes for trials in which targets appeared in the contralateral visual field (e.g., trials in the left visual hemifield for right hemisphere ROIs). We analyzed the trials in the left and right hemifield separately for 
A

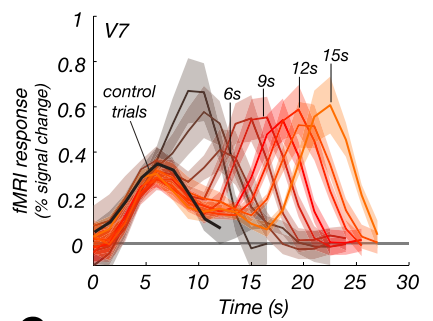

C

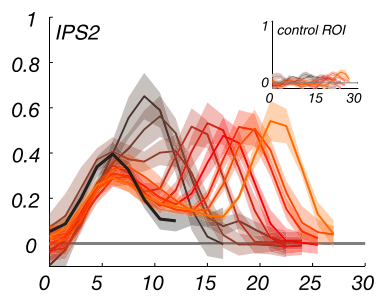

B

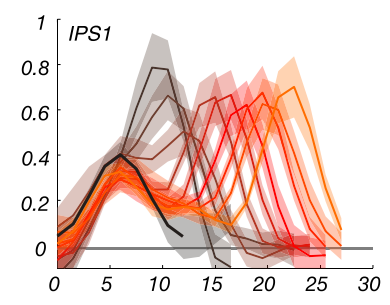

D

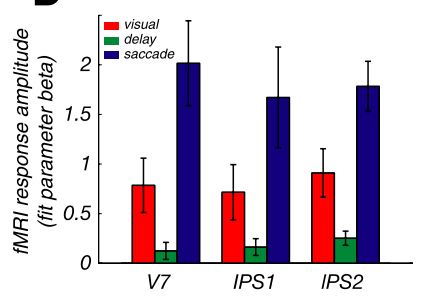

Figure 5. Average responses across subjects. $\boldsymbol{A}-\boldsymbol{D}$, fMRI responses as a function of time for different delay-period durations. Error bars indicate \pm 1 SEM across subjects. Each panel corresponds to a different cortical area: $\boldsymbol{A}$, V7; $\boldsymbol{B}$, IPS1; $\boldsymbol{C}$, IPS2; $\boldsymbol{C}$, inset, control IPS. $\boldsymbol{D}$, Response amplitudes estimated from the model fits and averaged across subjects. Red, Response to visual cue $(v)$; green, sustained delay-period response $(d)$; blue, response during saccade interval (s). Error bars indicate \pm 1 SEM.

each ROI and calculated a lateralization index from the estimated amplitude parameters (see Materials and Methods). Both responses to the presentation of the visual cue and sustained delayperiod activity showed substantial lateralization (Fig. 6). The estimated response amplitudes during the saccade interval were not significantly lateralized (Fig. 6). During that interval, however, subjects made a saccade to the target and also a saccade in the opposite direction back to fixation. Responses during the saccade interval would therefore be expected to include both components for ipsiversive and contraversive movements on every trial.

Which of the lateralized components (response to the visual cue or sustained delay-period activity) was responsible for the topographic maps that were used to define V7, IPS1, and IPS2 in the topographic mapping experiment? On the one hand, it is possible that the lateralized response to the brief cue dominated the topography measurements because the amplitude of the response to the visual cue was larger. On the other hand, although lower in amplitude, the sustained delay-period activity may have dominated because it was integrated by the hemodynamics over a longer time. To assess which of the factors dominated, we performed additional model calculations using the parameters from

the model fits obtained in this experiment. We first calculated the fMRI responses for ipsilateral and contralateral trials given the mean fit amplitude parameters for each ROI (V7, IPS1, and IPS2) across subjects (Table 1). The timing parameters were chosen to match those of the topography experiment: (1) an impulse at the beginning of the trial reflecting a transient cue response; (2) a sustained component lasting $3 \mathrm{~s}$ to model delay-period activity; and (3) an impulse after the delay period to model the saccaderelated response. Second, we calculated the area under the simulated response curves (i.e., the total response per trial) for the ipsilateral and contralateral fMRI responses and the resulting lateralization index (see Materials and Methods). Third, we then repeated these calculations assuming that (1) only the transient response to the visual cue or (2) only the sustained delay-period activity was lateralized. Finally, we used these results to calculate the proportion of the total lateralization accounted for by each of the two components. For the $3 \mathrm{~s}$ delay-period duration (used in the topographic mapping experiment), the lateralization of the cue outweighed that of the sustained delay period by a factor of $\sim 2$. For longer delay-period durations $(>7.5 \mathrm{~s})$, conversely, the lateralization of the delay-period activity contributed increasingly, outweighing the effect of the lateralized cue response. These results suggest that, for the short delay-period durations used in the topographic mapping experiments, the observed topography was mostly attributable to the lateralized responses to the visual cue.

\section{Discussion}

We used an event-related protocol to demonstrate sustained delay-period activity in topographically organized areas of human posterior parietal cortex. To unambiguously demonstrate that the activity was sustained, we varied the duration of the delay period. The fMRI responses increased at the beginning of each trial, after the presentation of the saccade target, and remained elevated for a variable amount of time depending on the duration of the delay period. At the end of the trial, we observed another transient response to the movement interval. We obtained similar results for each of three topographically organized cortical areas: V7, IPS1, and IPS2. The estimated amplitude of the delayperiod responses was $<30 \%$ of that elicited during the presentation of the visual cue. Both the cue and delay-period responses were lateralized, suggesting that both of these components contributed to the topography observed in the previous mapping experiments. Calculations based on the model fits indicated that, for short delay periods ( $3 \mathrm{~s})$, the lateralization of the cue responses was the dominant effect. A control region in the IPS just

Table 2. Statistical significance of response amplitudes

\begin{tabular}{|c|c|c|c|c|c|}
\hline Subject & Region of interest & $v$ & $d$ & $s$ & $\mathrm{DOF}$ \\
\hline \multirow[t]{3}{*}{1} & V7 & $20.44\left(<10^{-4}\right)$ & $10.90\left(<10^{-4}\right)$ & $20.36\left(<10^{-4}\right)$ & 31 \\
\hline & IPS1 & $15.50\left(<10^{-4}\right)$ & $10.78\left(<10^{-4}\right)$ & $15.91\left(<10^{-4}\right)$ & \\
\hline & IPS2 & $13.18\left(<10^{-4}\right)$ & $10.39\left(<10^{-4}\right)$ & $9.98\left(<10^{-4}\right)$ & \\
\hline \multirow[t]{3}{*}{2} & V7 & $8.69\left(<10^{-4}\right)$ & $-3.95(>0.1)$ & $22.98\left(<10^{-4}\right)$ & 31 \\
\hline & IPS1 & $7.46\left(<10^{-4}\right)$ & $-2.09(>0.1)$ & $15.60\left(<10^{-4}\right)$ & \\
\hline & IPS2 & $15.40\left(<10^{-4}\right)$ & $3.43(<0.01)$ & $19.62\left(<10^{-4}\right)$ & \\
\hline \multirow[t]{3}{*}{3} & V7 & $3.39(<0.05)$ & $7.58\left(<10^{-4}\right)$ & $6.87\left(<10^{-4}\right)$ & 27 \\
\hline & IPS1 & $4.07(<0.01)$ & $4.57\left(<10^{-4}\right)$ & $5.02\left(<10^{-4}\right)$ & \\
\hline & IPS2 & $3.41(<0.01)$ & $7.25\left(<10^{-4}\right)$ & $6.39\left(<10^{-4}\right)$ & \\
\hline \multirow[t]{3}{*}{4} & V7 & $1.33(>0.05)$ & $6.06\left(<10^{-4}\right)$ & $9.43\left(<10^{-4}\right)$ & 15 \\
\hline & IPS1 & $1.45(>0.05)$ & $6.55\left(<10^{-4}\right)$ & $9.29\left(<10^{-4}\right)$ & \\
\hline & IPS2 & $4.36(<0.01)$ & $14.93\left(<10^{-4}\right)$ & $10.59\left(<10^{-4}\right)$ & \\
\hline
\end{tabular}

$t$ statistic and $p$ values are listed for each cortical area in each subject. Entries in boldface indicate statistical significance at $p<0.05$, one-tailed $t$ test (hull hypothesis, mean of 0 ). DOF (degrees of freedom) $=n-1$, where $n$ is twice the number of scans (assuming that the measurements were statistically independent in the two hemispheres). 
A

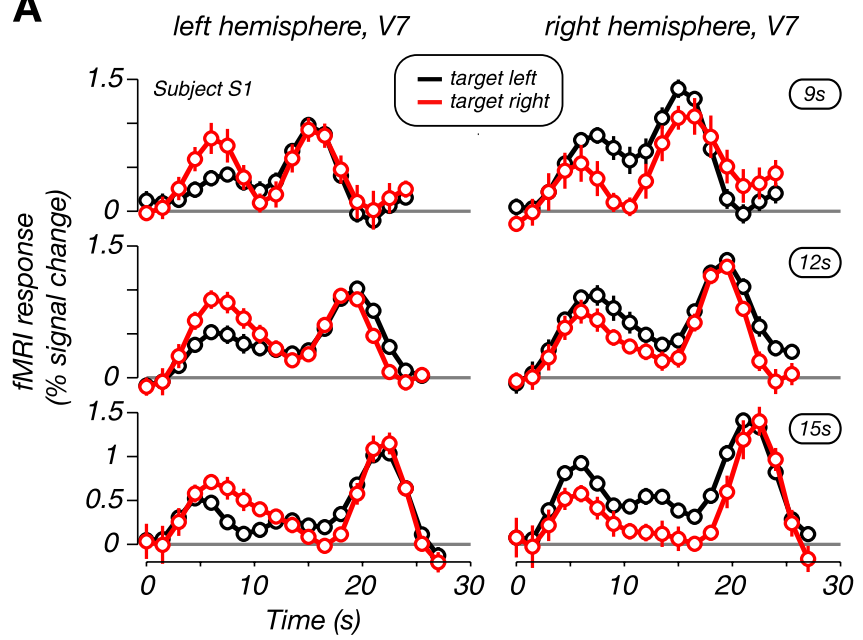

B

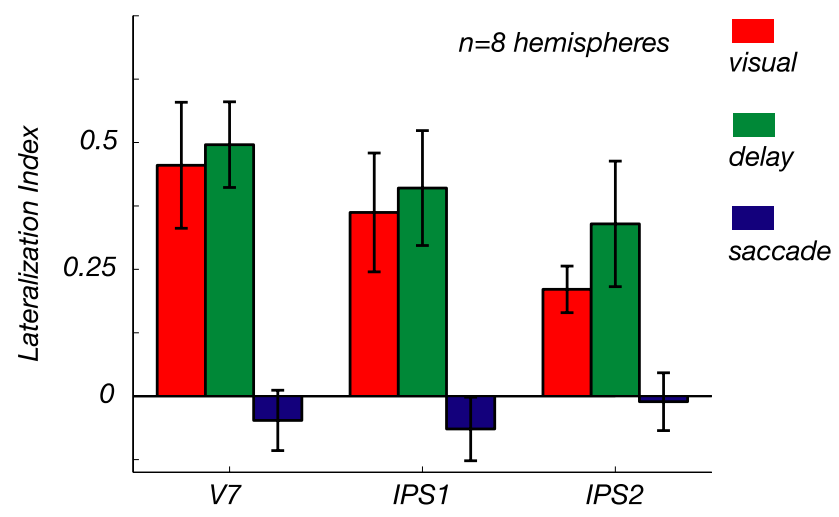

Figure 6. Lateralization of fMRI responses. $\boldsymbol{A}$, fMRI responses from the left and right hemisphere V7 for a subset of delay-period durations ( 9,12 , and $15 \mathrm{~s})$. Black lines, Trials presented in left visual field; red lines, trials in right visual field. Mean \pm SEM across trials. Responses to the visual cue and during the delay are larger for contralateral trials (red above black in the left column and black above red in the right column). The corresponding lateralization indices were as follows, for $v, d$, and s, respectively: $0.24,0.27,-0.19$ (left hemisphere V7); and 0.29, 0.48, 0.11 (right hemisphere V7). Note that the indices were calculated using the best-fit parameters estimated from all delay-period durations, not just the subset shown here. $\boldsymbol{B}$, Lateralization indices for V7, IPS1, and IPS2 (see Materials and Methods). Bars and error bars indicate mean and SEM across eight hemispheres.

lateral to these cortical areas did not respond during memoryguided saccades.

Posterior parietal cortex contains a number of separate and distinct cortical areas believed to perform different functions. Cortical areas have been identified with precision in macaque monkey parietal cortex based on a combination of physiological and anatomical measurements (Andersen et al., 1990a; Colby and Duhamel, 1991; Lewis and Van Essen, 2000a,b; Andersen and Buneo, 2002). Much of the research in macaque posterior parietal cortex has concentrated on the LIP, a region in the intraparietal sulcus known to play an important role in the planning and production of saccadic eye movements (Andersen and Buneo, 2002; Goldberg et al., 2002; Glimcher, 2003). A defining physiological signature of monkey LIP is that neurons in this area exhibit visual response fields, movement fields, and delay-period activity during memory-saccade tasks of the type we examined here (Gnadt and Andersen, 1988; Barash et al., 1991a,b). Neurons in this region of the brain respond when a sensory stimulus is presented, when attention is paid to a stimulus, while maintaining the mem- ory of a stimulus, while computing the sensory-motor transformation involved in making a movement to a stimulus, while maintaining the intention to make such a movement, and while computing the decision concerning which of two or more movements to make (for review, see Andersen and Buneo, 2002; Goldberg et al., 2002; Glimcher, 2003).

Neuroimaging studies of human posterior parietal cortex have revealed potentially homologous regions (for review, see Fink, 2005). The medial bank of the human IPS has been found to respond during manual reaching, whereas the lateral bank has been found to be selective for attention and eye movements (Rushworth et al., 2001; Astafiev et al., 2003; Connolly et al., 2003), similar to what has been reported for monkey areas LIP and PRR/medial intraparietal area (Snyder et al., 1997). Human posterior parietal cortex has been shown to exhibit some of the response properties that characterize monkey LIP. There are numerous reports of sustained activity in human posterior parietal cortex during a delay period before subjects make saccades (Connolly et al., 2002; Astafiev et al., 2003; Curtis et al., 2004; Curtis and D'Esposito, 2006). Human posterior parietal cortex also exhibits spatial updating after eye movements (Medendorp et al., 2003; Merriam et al., 2003), analogous to what has been observed in macaque LIP (Duhamel et al., 1992). An area in the ventral portion of the IPS has been found to respond to visual, auditory, and somatosensory motion (Bremmer et al., 2001), similar to what has been found in monkey ventral intraparietal area (Colby et al., 1993; Duhamel et al., 1998). An area in the anterior portion of the IPS has been found to respond during grasping or when subjects perform a crossmodal visuotactile delayed matchingto-sample task (Binkofski et al., 1998; Grefkes et al., 2002), analogous to what has been observed in monkey anterior intraparietal area (Sakata et al., 1995). Another area on the lateral bank of the IPS posterior to LIP in macaques has been reported to contain neurons sensitive to both monocular and binocular depth cues (Shikata et al., 1996; Tsutsui et al., 2002); a possible human homolog of this area CIP has also been proposed (Shikata et al., 2001). Activity in some areas of human posterior parietal cortex is modulated by eye position (DeSouza et al., 2000), similar to what has been found in monkeys (Andersen et al., 1985, 1990b; Stricanne et al., 1996; Snyder et al., 1998). Because we measured responses only during delayed saccades and because all three of the human cortical areas studied (V7, IPS1, and IPS2) exhibited similar results, and we cannot determine which of the human and macaque areas are most comparable with one another. The delay-period activity reported here might not be tied to specifically to saccades. Rather, it might be present during other tasks involving other (e.g., reaching) movements as well.

Progress in our understanding of posterior parietal function would be accelerated by enhancing our knowledge about the correspondence between human and macaque parietal cortical areas. Whereas single-unit electrophysiology in the macaque has focused on targeted and well defined cortical areas (such as LIP), this has not typically been the case with human fMRI studies that instead have usually revealed relatively large swaths of activity in a frontoparietal network of cortical areas. Specifically, Connolly et al. (2002) examined the responses in parietal and frontal cortex related to preparation of prosaccadic and anti-saccadic eye movements. They concluded that only the responses in frontal cortex, not parietal cortex, are directly related to the preparation of saccadic eye movements. Astafiev et al. (2003) reported the locations of statisti- 
cally significant responses in parietal and frontal cortex to "attending," "looking," and "pointing." They also reported potential correspondences of frontal and parietal areas in macaques and humans. The study by Curtis et al. (2004) compared delay-period activity during memory-guided saccade trials in which the metrics of the memory-guided saccade were or were not known during the delay. Both frontal eye fields (FEF) and intraparietal sulcus showed persistent delay-period activity in both conditions. However, activity was slightly increased in FEF and slightly decreased in parietal cortex when the metrics of the upcoming saccade were known before the delay. Following up on this finding, Curtis and D'Esposito (2006) specifically examined the effect of response selection on delay-period activity in the FEF and parietal cortex. They found that, once subjects selected a saccade among several possibilities, delay-period activity in FEF but not parietal cortex persisted throughout a long and variable retention interval until the memory-guided saccade was finally generated. The tasks in each of these studies were markedly different from ours, however, and the parietal areas revealed in most of these studies were anterior to V7, IPS1, and IPS2. The delay-period durations used in the studies by Medendorp et al. $(2003,2005)$ were very short $(1.25 \mathrm{~s})$, and their localization protocol only allowed them to establish lateralization rather than topographic organization. The Curtis and D'Esposito (2006) study was the only of these in which the duration of the delay-period interval was long and variable, as in our study, to disambiguate delay-period activity from sensory and motor responses. None of these previous studies, however, were aimed at quantifying delay-period responses in topographically localized areas of interest. Hence, none of these studies provided the necessary information to establish potential homologies between human and macaque parietal areas. A recent fMRI study provided a road map for the possible homologies between oculomotor areas in the monkey and human cortex by running exactly the same protocol in both species (Koyama et al., 2004); although comparable regions of cortex were activated, much more work remains to be done to identify and precisely localize the functionally homologous areas in different parts of the brain.

How do the responses during the delay period measured with fMRI compare with those reported in electrophysiological studies? Sustained activity in a delayed visually guided saccade task is the criterion by which many laboratories confirm the placement of their electrodes in the lateral bank of the IPS. Usually, however, only delay periods that would be considered short in our context $(<2000 \mathrm{~ms})$ are used in the screening of these cells, partly because this provides sufficient information for selecting neurons but also because it is difficult for the animals to perform the task with longer delays. In a study of response properties of 54 LIP neurons, Barash et al. (1991a) reported a ratio of $\sim 2: 1$ in the levels of relative activity during the "light-sensitive" and "memory" intervals (firing rates normalized to baseline) for what would be considered short delays here. Similarly, Paré and Wurtz (1997) reported the firing rates of macaque LIP neurons $(n=50)$ in both delayed visually guided and memory guided saccades, for short delays (500-1000 ms) (Paré and Wurtz, 1997, their Table 1). In their sample of neurons with significant delay-period activity, the responses to the visual cue were larger than that during the memory interval by a ratio of $\sim 2: 1$ (Paré and Wurtz, 2001).

The amplitude of the delay-period activity in the fMRI measurements we report here was smaller $(\sim 15-28 \%$ or be- tween $6: 1$ and 3.5:1) than that reported in the electrophysiology studies. There are several reasons that may account for the differences between these measurements by the two different techniques. First, although BOLD fMRI reflects the metabolic demand of neural activity, the precise relationship to neural firing rates is not known (Mathiesen et al., 1998; Lauritzen, 2001; Logothetis et al., 2001; Heeger and Ress, 2002; Logothetis and Wandell, 2004). It is possible that subthreshold changes in neural activity, invisible to single-unit recording, may be accompanied by substantial changes in $\mathrm{fMRI}$ response. In particular, the measured fMRI responses during the cue and saccade response periods in our experiment might reflect more subthreshold (feedback) modulation across the populations of neurons, which does not significantly affect the firing rates of individual neurons in LIP. This would then predict that the fMRI measurements underestimate the delay-period activity relative to the single-unit electrophysiology measurements, similar to what we observed. Second, sustained delayperiod activity is usually measured electrophysiologically in very short trials. Short delays are impractical for fMRI measurements of delay-period activity because of the temporal delay and dispersion of the hemodynamic response (Fig. 4). It is possible that delay-period activity in parietal neurons declines for longer delay-period durations ( $>8 \mathrm{~s}$ ). Currently, no published electrophysiology data from LIP neurons exists for memory-guided saccades with such long durations. Third, the electrophysiological results were based on a sample of cells that had been preselected because they showed significant delay-period activity. The fMRI measurements, conversely, may more accurately reflect the pooled activity over a whole population of neurons, some of which may exhibit strong delay-period activity, and others, which may be overlooked or undersampled in electrophysiology experiments exhibit only weak delay-period activity.

To conclude, the results we present here provide the first clear evidence that the topographically organized areas in human posterior parietal cortex (IPS1 and IPS2) show sustained, lateralized delay-period activity in memory-guided saccades, a defining feature of macaque areas such as LIP and PRR. This need not have been the case because there was no means for assessing delayperiod activity per se from the topographic mapping experiments. These areas are therefore potential homologs of monkey parietal regions.

\section{References}

Andersen RA, Buneo CA (2002) Intentional maps in posterior parietal cortex. Annu Rev Neurosci 25:189-220.

Andersen RA, Essick GK, Siegel RM (1985) Encoding of spatial location by posterior parietal neurons. Science 230:456-458.

Andersen RA, Asanuma C, Essick G, Siegel RM (1990a) Corticocortical connections of anatomically and physiologically defined subdivisions within the inferior parietal lobule. J Comp Neurol 296:65-113.

Andersen RA, Bracewell RM, Barash S, Gnadt JW, Fogassi L (1990b) Eye position effects on visual, memory, and saccade-related activity in areas LIP and 7a of macaque. J Neurosci 10:1176-1196.

Astafiev SV, Shulman GL, Stanley CM, Snyder AZ, Van Essen DC, Corbetta M (2003) Functional organization of human intraparietal and frontal cortex for attending, looking, and pointing. J Neurosci 23:4689-4699.

Barash S, Bracewell RM, Fogassi L, Gnadt JW, Andersen RA (1991a) Saccade-related activity in the lateral intraparietal area. I. temporal properties; comparison with area 7a. J Neurophysiol 66:1095-1108.

Barash S, Bracewell RM, Fogassi L, Gnadt JW, Andersen RA (1991b) Saccade-related activity in the lateral intraparietal area. II. Spatial properties. J Neurophysiol 66:1109-1124.

Binkofski F, Dohle C, Posse S, Stephan KM, Hefter H, Seitz RJ, Freund HJ 
(1998) Human anterior intraparietal area subserves prehension: a combined lesion and functional MRI activation study. Neurology 50:1253-1259.

Biswal B, Yetkin FZ, Haughton VM, Hyde JS (1995) Functional connectivity in the motor cortex of resting human brain using echo-planar MRI. Magn Reson Med 341:537-541.

Biswal B, Hudetz AG, Yetkin FZ, Haughton VM, Hyde JS (1997a) Hypercapnia reversibly suppresses low-frequency fluctuations in the human motor cortex during rest using echo-planar MRI. J Cereb Blood Flow Metab 17:301-308.

Biswal BB, Van Kylen J, Hyde JS (1997b) Simultaneous assessment of flow and bold signals in resting-state functional connectivity maps. NMR Biomed 10:165-170.

Bremmer F, Schlack A, Shah NJ, Zafiris O, Kubischik M, Hoffmann K, Zilles K, Fink GR (2001) Polymodal motion processing in posterior parietal and premotor cortex: a human fMRI study strongly implies equivalencies between humans and monkeys. Neuron 29:287-296.

Burock MA, Dale AM (2000) Estimation and detection of event-related fMRI signals with temporally correlated noise: a statistically efficient and unbiased approach. Hum Brain Mapp 11:249-260.

Burock MA, Buckner RL, Woldorff MG, Rosen BR, Dale AM (1998) Randomized event-related experimental designs allow for extremely rapid presentation rates using functional MRI. NeuroReport 9:3735-3739.

Colby CL, Duhamel JR (1991) Heterogeneity of extrastriate visual areas and multiple parietal areas in the macaque monkey. Neuropsychologia 29:517-537.

Colby CL, Duhamel JR, Goldberg ME (1993) Ventral intraparietal area of the macaque: anatomic location and visual response properties. J Neurophysiol 69:902-914.

Connolly JD, Goodale MA, Menon RS, Munoz DP (2002) Human fMRI evidence for the neural correlates of preparatory set. Nat Neurosci 5:1345-1352.

Connolly JD, Andersen RA, Goodale MA (2003) FMRI evidence for a "parietal reach region" in the human brain. Exp Brain Res 153:140-145.

Curtis CE, D'Esposito M (2003) Persistent activity in the prefrontal cortex during working memory. Trends Cogn Sci 7:415-423.

Curtis CE, D’Esposito M (2006) Selection and maintenance of saccade goals in the human frontal eye fields. J Neurophysiol, in press.

Curtis CE, Rao VY, D’Esposito M (2004) Maintenance of spatial and motor codes during oculomotor delayed response tasks. J Neurosci 24:3944-3952.

Dale AM (1999) Optimal experimental design for event-related fMRI. Hum Brain Mapp 83:109-114.

DeSouza JF, Dukelow SP, Gati JS, Menon RS, Andersen RA, Vilis T (2000) Eye position signal modulates a human parietal pointing region during memory-guided movements. J Neurosci 20:5835-5840.

DeYoe EA, Carman GJ, Bandettini P, Glickman S, Wieser J, Cox R, Miller D, Neitz J (1996) Mapping striate and extrastriate visual areas in human cerebral cortex. Proc Natl Acad Sci USA 93:2382-2386.

Duhamel JR, Colby CL, Goldberg ME (1992) The updating of the representation of visual space in parietal cortex by intended eye movements. Science 255:90-92.

Duhamel JR, Colby CL, Goldberg ME (1998) Ventral intraparietal area of the macaque: congruent visual and somatic response properties. J Neurophysiol 79:126-136.

Engel SA, Rumelhart DE, Wandell BA, Lee AT, Glover GH, Chichilnisky EJ, Shadlen MN (1994) fMRI of human visual cortex. Nature 369:525.

Engel SA, Glover GH, Wandell BA (1997) Retinotopic organization in human visual cortex and the spatial precision of functional MRI. Cereb Cortex 7:181-192.

Fink GR (2005) The functional organization of the intraparietal sulcus in humans and monkeys. J Anat 207:3-17.

Friston KJ, Frith CD, Liddle PF, Frackowiak RS (1991) Comparing functional (PET) images: the assessment of significant change. J Cereb Blood Flow Metab 11:690-699.

Friston K, Holmes A, Poline J, Grasby PJ, Williams SCR, Frackowiak R, Turner R (1995) Analysis of fMRI time series revisited. NeuroImage 2:45-53.

Glimcher PW (2003) The neurobiology of visual-saccadic decision making. Annu Rev Neurosci 26:133-179.

Glover GH (1999) Deconvolution of impulse response in event-related BOLD fMRI. NeuroImage 9:416-429.
Gnadt JW, Andersen RA (1988) Memory related motor planning activity in posterior parietal cortex of macaque. Exp Brain Res 70:216-220.

Goldberg ME, Bisley J, Powell KD, Gottlieb J, Kusunoki M (2002) The role of the lateral intraparietal area of the monkey in the generation of saccades and visuospatial attention. Ann NY Acad Sci 956:205-215.

Grefkes C, Weiss PH, Zilles K, Fink GR (2002) Crossmodal processing of object features in human anterior intraparietal cortex: an fMRI study implies equivalencies between humans and monkeys. Neuron 35:173-184.

Heeger DJ, Ress D (2002) What does fmri tell us about neuronal activity? Nat Rev Neurosci 3:142-151.

Jenkinson M, Bannister P, Brady J, Smith S (2002) Improved optimisation for the robust and accurate linear registration and motion correction of brain images. NeuroImage 17:825-841.

Jezzard P, Matthews PM, Smith SM, eds (2003) Functional magnetic resonance imaging: an introduction to methods. Oxford: Oxford UP.

Koyama M, Hasegawa I, Osada T, Adachi Y, Nakahara K, Miyashita Y (2004) Functional magnetic resonance imaging of macaque monkeys performing visually guided saccade tasks: comparison of cortical eye fields with humans. Neuron 41:795-807.

Larsson J (2001) Imaging vision: functional mapping of intermediate visual processes in man. $\mathrm{PhD}$ thesis, Karolinska Institutet.

Lauritzen M (2001) Relationship of spikes, synaptic activity, and local changes of cerebral blood flow. J Cereb Blood Flow Metab 21:1367-1383.

Lewis JW, Van Essen DC (2000a) Mapping of architectonic subdivisions in the macaque monkey, with emphasis on parieto-occipital cortex. J Comp Neurol 428:79-111.

Lewis JW, Van Essen DC (2000b) Corticocortical connections of visual, sensorimotor, and multimodal processing areas in the parietal lobe of the macaque monkey. J Comp Neurol 428:112-137.

Logothetis NK, Wandell BA (2004) Interpreting the bold signal. Annu Rev Physiol 66:735-769.

Logothetis NK, Pauls J, Augath M, Trinath T, Oeltermann A (2001) Neurophysiological investigation of the basis of the fmri signal. Nature 412:150-157.

Mathiesen C, Caesar K, Akgoren N, Lauritzen M (1998) Modification of activity-dependent increases of cerebral blood flow by excitatory synaptic activity and spikes in rat cerebellar cortex. J Physiol (Lond) 512:555-566.

Medendorp WP, Goltz HC, Vilis T, Crawford JD (2003) Gaze-centered updating of visual space in human parietal cortex. J Neurosci 23:6209-6214.

Medendorp WP, Goltz HC, Vilis T (2005) Remapping the remembered target location for anti-saccades in human posterior parietal cortex. J Neurophysiol 94:734-740.

Merriam EP, Genovese CR, Colby CL (2003) Spatial updating in human parietal cortex. Neuron 39:361-373.

Paré M, Wurtz RH (1997) Monkey posterior parietal cortex neurons antidromically activated from superior colliculus. J Neurophysiol 78:3493-3497.

Paré M, Wurtz RH (2001) Progression in neuronal processing for saccadic eye movements from parietal cortex area LIP to superior colliculus. J Neurophysiol 85:2545-2562.

Passingham D, Sakai K (2004) The prefrontal cortex and working memory: physiology and brain imaging. Curr Opin Neurobiol 14:163-168.

Purdon P, Weisskoff R (1998) Effect of temporal autocorrelation due to physiological noise and stimulus paradigm on voxel-level false-positive rates in fMRI. Hum Brain Mapp 61:239-249.

Rushworth MF, Paus T, Sipila PK (2001) Attention systems and the organization of the human parietal cortex. J Neurosci 21:5262-5271.

Sakata H, Taira M, Murata A, Mine S (1995) Neural mechanisms of visual guidance of hand action in the parietal cortex of the monkey. Cereb Cortex 5:429-438.

Schluppeck D, Glimcher PW, Heeger DJ (2005) Topographic organization for delayed saccades in human posterior parietal cortex. J Neurophysiol 94:1372-1384.

Sereno MI, Dale AM, Reppas JB, Kwong KK, Belliveau JW, Brady TJ, Rosen BR, Tootell RB (1995) Borders of multiple visual areas in humans revealed by functional magnetic resonance imaging. Science 268:889-893.

Sereno MI, Pitzalis S, Martinez A (2001) Mapping of contralateral space in 
retinotopic coordinates by a parietal cortical area in humans. Science 294:1350-1354.

Shikata E, Tanaka Y, Nakamura H, Taira M, Sakata H (1996) Selectivity of the parietal visual neurones in $3 \mathrm{~d}$ orientation of surface of stereoscopic stimuli. NeuroReport 7:2389-2394.

Shikata E, Hamzei F, Glauche V, Knab R, Dettmers C, Weiller C, Buchel C (2001) Surface orientation discrimination activates caudal and anterior intraparietal sulcus in humans: an event-related fMRI study. J Neurophysiol 85:1309-1314.

Smith AM, Lewis BK, Ruttimann UE, Ye FQ, Sinnwell TM, Yang Y, Duyn JH, Frank JA (1999) Investigation of low frequency drift in fMRI signal. NeuroImage 93:526-533.

Snyder LH, Batista AP, Andersen RA (1997) Coding of intention in the posterior parietal cortex. Nature 386:167-170.

Snyder LH, Grieve KL, Brotchie P, Andersen RA (1998) Separate body- and world-referenced representations of visual space in parietal cortex. Nature 394:887-891.
Srimal R, Lauer ME, Curtis CE (2005) Persistent activity in human frontal and parietal cortex during maintenance of spatial cues. Soc Neurosci Abstr 31:65.19.

Stricanne B, Andersen RA, Mazzoni P (1996) Eye-centered, head-centered, and intermediate coding of remembered sound locations in area LIP. J Neurophysiol 76:2071-2076.

Tsutsui K-I, Sakata H, Naganuma T, Taira M (2002) Neural correlates for perception of $3 \mathrm{~d}$ surface orientation from texture gradient. Science 298:409-412.

White JM, Sparks DL, Stanford TR (1994) Saccades to remembered target locations: an analysis of systematic and variable errors. Vision Res 34:79-92.

Worsley KJ, Evans AC, Marrett S, Neelin P (1992) A three-dimensional statistical analysis for CBF activation studies in human brain. J Cereb Blood Flow Metab 12:900-918.

Zarahn E, Aguirre GK, D’Esposito M (1997) Empirical analysis of BOLD fMRI statistics. I. Spatially unsmoothed data collected under nullhypothesis conditions. NeuroImage 5:179-197. 